\title{
Capacitação de profissionais da saúde na área de saúde auditiva: revisão sistemática
}

\section{The training of health professionals in hearing health: a systematic review}

\author{
Tatiana Mendes de Melo ${ }^{1}$, Kátia de Freitas Alvarenga ${ }^{2}$
}

\begin{abstract}
RESUMO
O objetivo deste trabalho foi avaliar a capacitação de profissionais da saúde sobre saúde auditiva por meio da revisão sistemática. O levantamento bibliográfico foi conduzido em base eletrônica de dados (Lilacs, SciELO, Medline, Scopus, Embase, Science Direct Online e Web of Science), revistas online da área de audiologia, anais de congressos nacionais e bibliotecas de universidades brasileiras, com busca bibliográfica padronizada, utilizando-se palavras-chave específicas procurando todos os resumos de estudos pertinentes à questão proposta. Para a seleção e avaliação dos estudos científicos levantados na busca foram estabelecidos critérios, contemplando os aspectos: tipo de estudo, participantes, intervenção adotada e avaliação dos resultados. Dos títulos obtidos a partir desta busca foram selecionados apenas aqueles cujo resumo ou corpo do artigo tivesse relação com o objeto do presente estudo. Após a leitura do texto completo, os mesmos foram resenhados de acordo com ficha protocolar e classificados com relação ao nível de evidência e grau de recomendação. Como resultado cinco estudos foram incluídos na revisão sistemática. Existe uma escassez de estudos com este enfoque, contudo os resultados obtidos demonstraram que a capacitação dos profissionais em saúde auditiva é efetiva, aumentando o conhecimento dos mesmos sobre o tema e, consequentemente, identificando e encaminhando os indivíduos com sinais de distúrbios auditivos para os serviços de referência.
\end{abstract}

Descritores: Audição; Deficiência auditiva; Capacitação de recursos humanos em saúde; Capacitação em serviço; Pessoal de saúde/ educação

\section{INTRODUÇÃO}

$\mathrm{O}$ atendimento ao deficiente auditivo foi incorporado pelo Sistema Único de Saúde (SUS) no início da década de 90 e, desde então, houve a publicação de algumas Portarias que, embora fossem de extrema importância para o acesso da população ao tratamento, não englobavam todos os procedimentos que visam boas práticas de atenção e cuidado à saúde auditiva.

Em 2004, o Ministério da Saúde, após sentir a necessidade de rever no país a efetividade dos procedimentos de concessão de aparelho de amplificação sonora individual (AASI), instituiu a Política Nacional de Atenção à Saúde Auditiva por meio da Portaria GM/MS n 2.073 de 28/09/2004 ${ }^{(1)}$.

Esta Política permitiu um grande avanço no aprimoramento

Trabalho realizado no Departamento de Fonoaudiologia da Faculdade de Odontologia de Bauru da Universidade de São Paulo - USP - Bauru (SP), Brasil.

(1) Mestre, Fonoaudióloga da Faculdade de Odontologia de Bauru da Universidade de São Paulo - FOB-USP - Bauru (SP), Brasil.

(2) Doutora, Professora Associada do Departamento de Fonoaudiologia da Faculdade de Odontologia de Bauru da Universidade de São Paulo - FOBUSP - Bauru (SP), Brasil.

Endereço para correspondência: Kátia de Freitas Alvarenga. Al. Dr Octávio Pinheiro Brisolla, 9-75, Bauru - SP, CEP: 17012-101.

E-mail: katialv@fob.usp.br

Recebido em 8/10/2008; Aceito em: 14/1/2009 das ações de saúde auditiva do SUS, na medida em que propôs a organização de uma rede hierarquizada, regionalizada e integrada na atenção básica, média e alta complexidade. Desta maneira, o tratamento da deficiência auditiva foi garantido como um todo, contemplando não só o diagnóstico e adaptação do AASI, mas também a promoção da saúde auditiva, bem como a reabilitação tanto de adultos como de crianças.

O Anexo I da Portaria SAS no. 587, no qual aponta as normas de atendimento na Rede de Atenção à Saúde Auditiva, especifica que as ações de saúde auditiva na atenção básica compreendem o trabalho voltado para promoção da saúde auditiva, prevenção e identificação precoce da alteração auditiva, por meio de ações informativas, educativas e de orientação familiar, e encaminhamento, quando necessário, para os serviços de saúde auditiva de referência. Contudo, as ações de atenção básica não são executadas pelo fonoaudiólogo e nem pelos médicos otorrinolaringologistas, por não fazerem parte das equipes de Saúde da Família, locus desta atenção ${ }^{(2)}$.

Ao considerar que atualmente, 87 milhões de brasileiros são acompanhados por mais de 28 mil equipes de Saúde da Família, presentes em $92 \%$ dos municípios ${ }^{(3)} \mathrm{e}$ a atenção básica seria a porta de acesso aos serviços de saúde auditiva, estes profissionais devem estar devidamente capacitados para as ações em saúde auditiva, a fim de proporcionar maior resolutibilidade nesta área. 
Em consonância à problemática exposta, o objetivo do presente estudo foi avaliar a capacitação de profissionais da saúde sobre saúde auditiva por meio da revisão sistemática da literatura.

\section{REVISÃO DA LITERATURA}

Considerando que a revisão sistemática é realizada a partir da formulação de perguntas específicas que direcionam a busca das publicações, a pergunta da investigação do presente estudo foi: "Qual a efetividade da capacitação de profissionais da saúde, para atuarem na área de saúde auditiva?".

\section{Critérios de seleção dos estudos}

Para a seleção e avaliação dos estudos científicos levantados na busca eletrônica foram estabelecidos critérios, contemplando os aspectos: tipo de estudos, participantes, a intervenção adotada e a avaliação dos resultados.

\section{Tipo de estudos}

Os artigos selecionados para análise foram os que apresentavam nível de evidência 1 - revisões sistemáticas e meta análises; 2 - estudos controlados randomizados e 3 - estudos de intervenção não randomizados, conforme adaptado para a área da Audiologia ${ }^{(4)}$. Vale ressaltar que, de acordo com os princípios da revisão sistemática, apenas estudos com níveis de evidência 1 e 2 devem ser selecionados. Contudo, caso este critério fosse adotado, muitas informações relevantes poderiam ser perdidas, visto que na Audiologia poucos estudos apresentam este desenho, revisões sistemáticas e estudos controlados randomizados.

\section{Participantes}

Para que o estudo fosse incluído na revisão sistemática, necessariamente teria que ter sido desenvolvido com profissionais da saúde, independente da área de atuação e/ou formação acadêmica.

\section{Intervenção}

A forma como a capacitação foi realizada não foi um critério de seleção estabelecido para esta revisão sistemática, sendo analisados os estudos que propuseram, desde a obtenção do conhecimento pelos participantes de forma informal, tal como a utilização de material impresso, como também intervenções formais, por meio da educação permanente e/ou continuada.

\section{Avaliação dos resultados}

Para a avaliação da capacitação dos profissionais da saúde, considerou-se tanto o impacto na atitude do profissional junto à comunidade, após receber as informações sobre saúde auditiva, como também a utilização de questionários e formas afins de avaliação dos resultados.

\section{Localização dos estudos}

\section{Descritores}

Para o levantamento dos descritores, utilizou-se o voca- bulário estruturado e trilíngue - Descritores em Ciências da Saúde (DeCS), criado pela Bireme para uso na indexação de artigos de revistas científicas, livros, anais de congressos, relatórios técnicos e outros tipos de materiais, assim como, para ser usado na pesquisa e recuperação de assuntos da literatura científica nas bases de dados.

O Quadro 1 apresenta as estratégias de busca utilizadas para o levantamento bibliográfico.

Quadro 1. Estratégias de busca (descritores) utilizadas no presente estudo

\begin{tabular}{|l|l|}
\hline \multicolumn{2}{|c|}{ Estratégia de busca } \\
\hline Descritores em Inglês & Descritores em Português \\
\hline Hearing and training & Audição and capacitação \\
\hline $\begin{array}{l}\text { Meta-analysis and hearing and } \\
\text { training }\end{array}$ & $\begin{array}{l}\text { Metanálise and audição and } \\
\text { capacitação }\end{array}$ \\
\hline Hearing loss and training & Perda auditiva and capacitação \\
\hline $\begin{array}{l}\text { Meta-analysis and hearing loss } \\
\text { and training }\end{array}$ & $\begin{array}{l}\text { Metanálise and perda auditiva } \\
\text { and capacitação }\end{array}$ \\
\hline
\end{tabular}

\section{Fonte de estudo}

No presente estudo, o levantamento bibliográfico foi conduzido em Bases de dados eletrônicas, revistas online da área de audiologia, anais de congressos nacionais e bibliotecas de universidades brasileiras.

\section{Bases de dados eletrônicas}

Inicialmente, foi realizado um levantamento da literatura a fim de averiguar a existência de trabalhos desenvolvidos com semelhante enfoque ao proposto no presente estudo. Para tanto foi realizada a pesquisa no Portal Cochrane da Biblioteca Virtual em Saúde (BVS) disponível no endereço: http://cochrane. bvsalud.org/portal/php/index.php?lang=pt. A partir desta busca verificou-se a inexistência de estudos de revisão sistemática ou indexação de pesquisas que focaram a capacitação de profissionais de saúde na área da saúde auditiva.

Desta forma, realizou-se o levantamento bibliográfico em bases de dados de acesso público, tais como: Lilacs, Medline e SciELO; e em bases de dados de acesso restrito, tais como: Scopus, Embase, Science Direct Online e Web of Science. Não houve restrição no ano de publicação, ou seja, foram analisados os estudos publicados até outubro de 2007. A partir desta data foi utilizada a opção "alerta" oferecida pelas bases eletrônicas, que se trata de uma ferramenta que permite ao pesquisador receber informações semanais ou mensais, conforme a sua escolha, sobre a publicação de novos estudos relacionados à estratégia de busca utilizada na base de dados.

\section{Base de dados de instituições de ensino superior}

Como o Brasil apresenta inúmeras Instituições de ensino superior, o levantamento de dados em bases de bibliotecas restringiu-se às Instituições de ensino superior do Estado de São Paulo, de caráter público, que contemplasse os cursos de Fonoaudiologia e Saúde Pública. Além destas, o levantamento bibliográfico também foi realizado na base de dados das Instituições públicas ou privadas, que oferecem cursos de Pós-graduação Strictu-senso em Fonoaudiologia ou Distúrbios 
da Comunicação Humana, de acordo com a Coordenação de Aperfeiçoamento de Pessoal de Nível Superior (CAPES). Desta forma, o levantamento bibliográfico foi realizado na base de dados da Universidade de São Paulo (USP), Universidade de Estadual de Campinas (UNICAMP), Universidade Estadual Paulista Júlio de Mesquita Filho (UNESP), Universidade Federal de São Paulo (UNIFESP); Pontifícia Universidade Católica de São Paulo (PUC-SP), Universidade Tuiuti do Paraná (UTP), Universidade Federal do Ceará (UFC), Universidade Estadual do Ceará (UCE), Universidade Federal de Minas Gerais (UFMG), Universidade Federal de Santa Maria (UFSM), Universidade Federal de Santa Catarina (UFSC), Fundação Oswaldo Cruz (FIOCRUZ) e Centro de Pesquisa Aggeu Magalhães/Fiocruz/PE (NESC/CpqAM).

\section{Anais de congressos}

Como há uma gama extensa de eventos científicos oferecidos e o levantamento de dados em anais necessita ser realizado de forma manual, demandando maior tempo, resolveu-se considerar os trabalhos científicos apresentados em Congressos nacionais de maior impacto na Audiologia, isto é, o Encontro Internacional de Audiologia e o Congresso Brasileiro de Fonoaudiologia dos últimos 10 anos (1997 a 2007).

Foram levantados, inicialmente, 14 trabalhos potencialmente relevantes para o presente estudo, sendo 12 referentes ao Encontro Internacional de Audiologia e dois referentes ao Congresso Brasileiro de Fonoaudiologia.

\section{Avaliação e seleção dos estudos}

Na busca, foram avaliados e selecionados apenas os estudos cujo título, resumo ou corpo do artigo tivessem relação com o objeto do presente estudo. Após a seleção dos resumos de estudos encontrados, pertinentes à questão proposta, foi realizada a recuperação dos artigos em texto completo.

Os dados de cada um dos artigos potencialmente relevantes para a revisão sistemática foram coletados por meio de uma ficha protocolar contendo: Critérios de elegibilidade do estudo, métodos utilizados, características dos pacientes incluídos no estudo; tipo de intervenções realizadas no estudo, desfechos mensurados e resultados obtidos.

\section{Análise dos dados}

Após a leitura do texto completo, os mesmos foram resenhados de acordo com a ficha protocolar e classificados de acordo com o tipo de pesquisa clínica, bem como com nível de evidência e grau de recomendação, conforme adaptados ${ }^{(4)}$ para o presente estudo.

\section{RESULTADOS OBTIDOS}

\section{Seleção dos estudos potencialmente relevantes para a revisão sistemática}

\section{Bases de dados eletrônicas}

Ao final do levantamento foram selecionados 31 estudos, porém foram excluídos os artigos duplicados nas bases de dados, totalizando assim 16 estudos potencialmente relevantes para a revisão sistemática.

Além dos 16 estudos relevantes selecionados para análise da revisão sistemática, dois outros artigos científicos de revista online não indexada em base de dados eletrônica, mas de importância científica na área de audiologia, foram adicionados por apresentarem relevância científica para o objeto do presente estudo.

\section{Anais de congressos}

Foram levantados, inicialmente, 14 trabalhos potencialmente relevantes para o presente estudo, sendo 12 referentes ao Encontro Internacional de Audiologia e dois referentes ao Congresso Brasileiro de Fonoaudiologia.

Bibliotecas de cursos de pós-graduação em áreas relacionadas à Fonoaudiologia

Nas bases de dados das bibliotecas de Instituições que apresentam cursos de Pós-graduação em áreas relacionadas à Fonoaudiologia, utilizou-se a mesma estratégia de busca descrita anteriormente. Nesta busca, não foi levantado nenhum trabalho de pós-graduação referente ao objeto do presente estudo.

\section{Análise dos estudos selecionados}

\section{Bases de dados eletrônicas}

Dos 18 estudos levantados, apenas cinco foram selecionados para a revisão sistemática após a leitura dos mesmos na íntegra. A exclusão dos demais artigos da análise deveu-se ao tipo de estudo realizado, isto é, cinco estudos foram excluídos por serem estudos transversais e que tinham por objetivo investigar quais os tópicos necessários para programas de capacitação de profissionais de saúde; os outros oito estudos foram excluídos por serem estudos de opinião de especialistas sobre a importância de programas de capacitação para profissionais da saúde. O Anexo 1 provê as referências bibliográficas dos estudos não selecionados para a revisão sistemática da literatura científica.

Dos cinco estudos selecionados para a revisão sistemática, três apresentaram nível de qualidade dois e os demais apresentaram nível de qualidade três. As referências bibliográficas dos estudos selecionados encontram-se no Anexo 2.

A Figura 1 apresenta uma síntese do processo de obtenção dos artigos selecionados para a revisão sistemática da literatura.

\section{Anais de congressos}

Dos 14 estudos potencialmente relevantes, nenhum deles foi selecionado para a revisão sistemática após a leitura dos mesmos na íntegra. A exclusão dos trabalhos deveu-se ao tipo de estudo realizado, isto é, três trabalhos foram excluídos por serem estudos descritivos (descrição dos tópicos abordados em cursos de capacitação); oito estudos foram excluídos por serem estudos transversais e que tinham por objetivo investigar quais os tópicos necessários para programas de capacitação de profissionais de saúde e os outros três estudos foram excluídos por apresentarem na casuística, profissionais que não contemplavam os critérios pré-estabelecidos para inclusão 


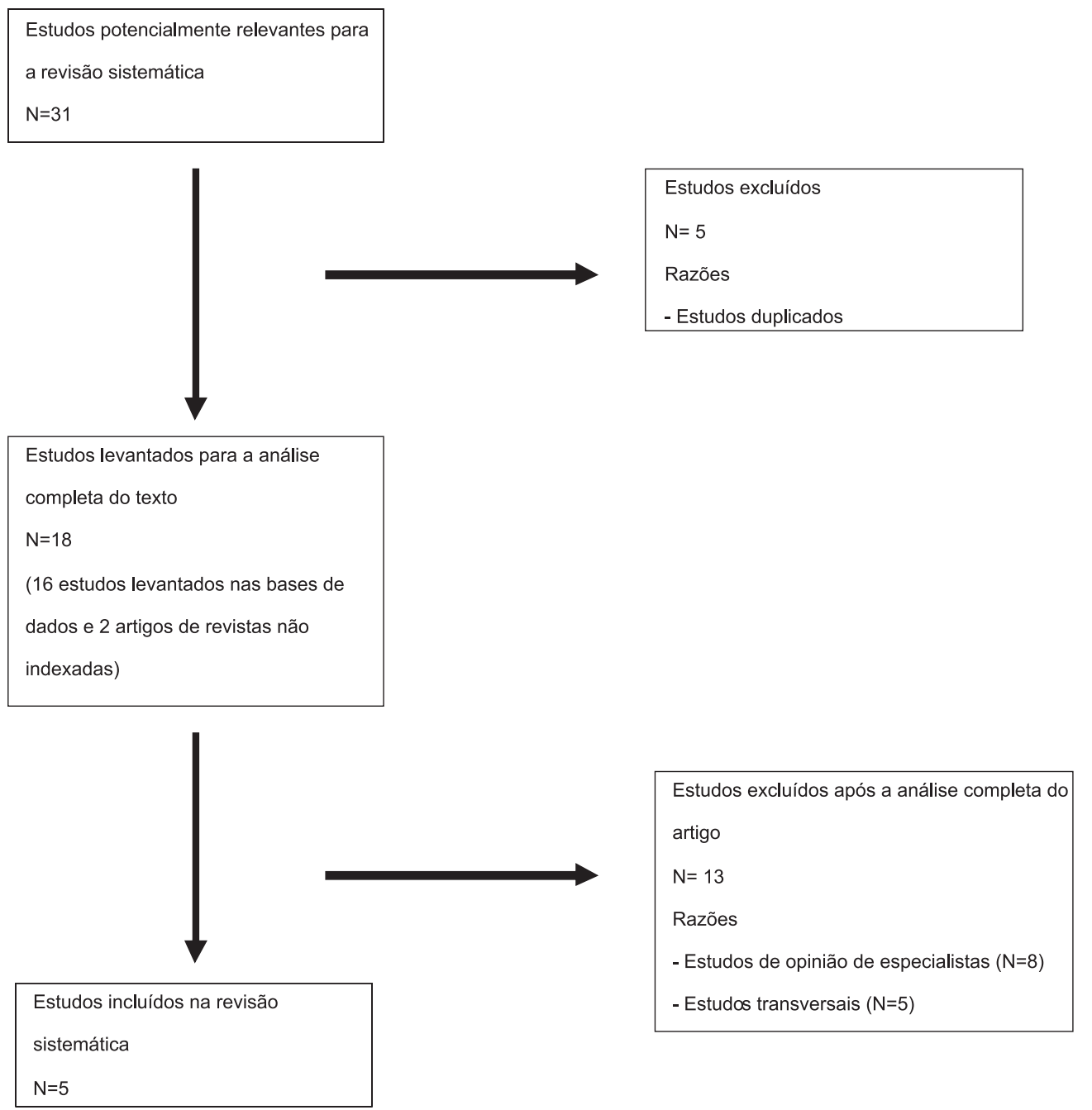

Figura 1. Síntese do processo de obtenção dos artigos selecionados para a revisão sistemática da literatura

dos estudos, ou seja, os participantes da capacitação não eram profissionais da área da saúde.

\section{DISCUSSÃO}

Desde a sua criação, o Programa de Saúde da Família vem se estendendo por todo o território nacional. Em dezembro de 1999, existiam 4.114 equipes de Saúde da Família atuando em 1.646 municípios, que representam 29,9\% dos municípios brasileiros. Atualmente, 87 milhões de brasileiros são acompanhados por mais de 28 mil equipes, presentes em $92 \%$ dos municípios $^{(3)}$.

Ao considerar que o Ministério da Saúde elegeu a Saúde da Família como o pilar para reorganização do modelo assistencial no Brasil e que a educação permanente é concebida como um importante instrumento para a consolidação do SUS, existe grande preocupação em garantir a capacitação desses profissionais nas diversas áreas da saúde.

O número expressivo da cobertura assistencial proporcionado pelas equipes de Saúde da Família em escala nacional também pode ter impacto na Rede de Atenção à Saúde Auditiva, uma vez que, quando capacitados de forma adequada, tais profissionais podem atuar em ações de promoção à saúde auditiva junto à comunidade nos diferentes ciclos de vida: gestantes, recém-nascidos, pré-escolares, escolares, jovens, trabalhadores e idosos.

Atualmente, a Política Nacional de Educação Permanente em Saúde ${ }^{(5)}$, apresenta como uma das atividades, mas não realizada de maneira compulsória, a formação em Atenção Integral às Doenças Prevalentes na Infância (AIDPI). O objetivo é o de abordar o conjunto de doenças de maior predominância na infância que demandam maior encaminhamento para os serviços de saúde. Nesta atividade de capacitação é abordado o tópico "como avaliar e classificar os problemas de ouvido", auxiliando o profissional a identificar sinais de otite. Todavia, este único aspecto aborda superficialmente o problema auditivo e não contempla todo universo referente à deficiência auditiva. Assim, para que os profissionais da Saúde da Família possam atuar na promoção da saúde auditiva de forma efetiva, evitando a ocorrência ou minimizando as consequências da deficiência auditiva, faz-se necessária a capacitação dos mesmos nesta área específica.

Para tanto, é importante considerar experiências anteriores na capacitação de profissionais da área da saúde em saúde auditiva, a fim de subsidiar práticas baseadas em evidências científicas. 
Após o processo da busca bibliográfica e a análise dos artigos potencialmente relevantes para a revisão sistemática, foram selecionados 18 estudos para a revisão sistemática da avaliação da capacitação de profissionais da saúde sobre saúde auditiva. Destes, apenas cinco estudos foram selecionados para a revisão sistemática.

Tal achado torna-se preocupante, uma vez que muito tem se discutido sobre a importância de capacitar profissionais de saúde, na área de saúde auditiva, para atuarem em prevenção; contudo, poucos estudos têm sido desenvolvidos com o intuito de demonstrar realmente a efetividade desta ação.

Outro aspecto importante refere-se ao fato de que os estudos na área de audiologia com semelhante enfoque deste trabalho apresentavam como desenho metodológico de pesquisa, na maioria das vezes, estudos descritivos e de opinião de especialistas, ou seja, apresentam nível de evidência baixa para considerar a contribuição dos resultados frente à decisão de realizar determinada intervenção ${ }^{(4)}$.

Dos estudos selecionados para a revisão sistemática, a maioria apresentava desenho metodológico do tipo estudo clínico aleatório ${ }^{(6-8)}$ e tiveram como participantes médicos, enfermeiros, agentes de saúde, psicólogos e cuidadores ${ }^{(6-10)}$.

A intervenção variou desde o uso de materiais impressos ${ }^{(7)}$ para capacitação até intervenções formais, como cursos de capacitação e/ou educação continuada ${ }^{(6,8-10)}$. Os resultados obtidos foram mensurados por meio de questionários ${ }^{(6,10)}$, aumento na taxa de encaminhamento aos serviços de saúde auditiva ${ }^{(7-8)}$ e concordância entre as observações clínicas do profissional especializado e do profissional capacitado ${ }^{(9)}$.

Após a análise dos estudos, foi possível observar consenso de que a capacitação de profissionais de saúde para atuarem em saúde auditiva leva a um impacto positivo, observado na mudança de comportamento do profissional e gestores públicos, tanto no conhecimento sobre o tema, quanto no acesso dos deficientes auditivos nos serviços de audiologia. Porém, os trabalhos analisados não evidenciam indicadores que possam ser descritos como essenciais para um curso de capacitação, uma vez que a definição dos conteúdos a serem ministrados na atividade deve levar em consideração as particularidades e realidade de cada região, para que seja um aprendizado com aplicabilidade na atuação dos profissionais junto à comunidade.

\section{COMENTÁRIOS FINAIS}

Após a extensa revisão da literatura nacional e internacional foi possível concluir que, desde o primeiro estudo desenvolvido focando a capacitação dos profissionais da área da saúde em saúde auditiva, realizado no início da década de 90, o que se observam subsequente na literatura específica, em sua maioria, são publicações voltadas a demonstrar o nível de conhecimento dos profissionais da saúde na área da audição e a necessidade de capacitação dos mesmos, sendo este último aspecto, um consenso entre os estudos analisados.

A literatura é escassa quando considerados os estudos desenvolvidos com o objetivo de validar programas de capacitação, assim como, demonstrar a mudança de comportamento dos profissionais da saúde frente à saúde auditiva da população após a introdução dos conceitos desta área específica na sua formação básica.

Contudo, os estudos pertinentes à proposta da revisão sistemática da literatura demonstraram que a capacitação dos profissionais em saúde auditiva é efetiva para a identificação da deficiência auditiva, aumentando o conhecimento dos profissionais sobre o tema e, consequentemente, identificando e encaminhando os indivíduos com sinais de distúrbio auditivo para os serviços de referência.

Na Portaria SAS no 587 de 2004, a capacitação das equipes de atenção básica, por meio do apoio técnico das equipes dos serviços de saúde auditiva na média e alta complexidade, é uma normativa. Assim, a análise das experiências prévias em capacitação de profissionais de saúde na área de saúde auditiva descritas na literatura é de extrema importância para subsidiar, a partir de evidências científicas, a incorporação de programas de capacitação junto às equipes de Saúde da Família e, consequentemente, o fortalecimento da rede de Atenção à Saúde Auditiva proposta nesta Portaria.

Após quatro anos da instauração da Política Nacional de Atenção à Saúde Auditiva, o Brasil apresenta 128 serviços habilitados em território nacional. Assim, para que as equipes de Saúde da Família atuem em saúde auditiva, devem considerar a existência de serviços credenciados em sua região, para que suas ações possam envolver não apenas a prevenção da alteração auditiva, mas também as diversas etapas de um processo de intervenção na deficiência auditiva.

\begin{abstract}
The aim of this study was to evaluate the training of health professionals regarding hearing health, through a systematic literature review. The bibliographic search was carried out in scientific database (Lilacs, SciELO, Medline, Scopus, Embase, Science Direct Online and Web of Science), online journals on audiology, proceedings from Brazilian conventions, and libraries from Brazilian universities, using specific keywords in order to find all the possible studies matching the proposed question. The studies that matched these criteria were selected and evaluated according to the following aspects: type of study, participants, intervention adopted and outcomes evaluation. After reading the selected manuscripts, they were summarized in a protocol and classified according to level of evidence and recommendation degree. Five studies were included in the systematic review. The results showed a shortage of researches that emphasize this topic, but the analysis of the articles demonstrated that the training of health professionals in hearing health is effective, increasing their knowledge on the theme and, consequently, enabling them to identify patients at risk for hearing loss and to refer these individuals to specific evaluation.
\end{abstract}

Keywords: Hearing; Hearing loss; Health human resource training; Inservice training; Health personnel/education 


\section{REFERÊNCIAS}

1. Brasil. Portaria GM/MS no 2.073 de 28 de setembro de 2004. Institui a Política Nacional de Atenção à Saúde Auditiva. Diário Oficial da União. 29 set 2004; Seção 1: 34.

2. Miranda GMD, Queiroga BAM, Lessa FJD, Leal MC, Caldas Neto SS. Diagnóstico da deficiência auditiva em Pernambuco: oferta de serviços de média complexidade - 2003. Rev Bras Otorrinolaringol. 2006;72(5):581-6.

3. Brasil. Ministério da Saúde. Secretaria-Executiva. Mais saúde: direito de todos: 2008 - 2011. 2a ed. Brasília: Ministério da Saúde; c2008. 100 p.

4. Cox RM. Waiting for evidence-based practice for your hearing aid fittings? It's here! Hear J. 2004;57(8):10-7.

5. Brasil. Ministério da Saúde. Portaria GM/MS no 198 de 13 de fevereiro de 2004. Institui a Política nacional de educação permanente em saúde como estratégia do Sistema Único de Saúde para formação e desenvolvimento de trabalhadores para o setor e dá outras providências. Diário Oficial da Republica Federativa do Brasil. 29 fev 2004; Seção 1: 24.
6. McMillan L, Bunning K, Pring T. The development and evaluation of deaf awareness training course for support staff. J Appl Res Intellect Disabil. 2001;13(4):283-91.

7. Bennett K, Haggard M, Churchill R, Wood S. Improving referrals for glue ear from primary care: are multiple interventions better than one alone? J Health Serv Res Policy. 2001;6(3):139-44.

8. Kochkin S. BHI physician program found to increase use of hearing healthcare. The Hearing Journal. 2004;57(8):27-9.

9. Gloria-Cruz TL, Chiong CM, Chan AL, Llanes EG, Reyes-Quintos MR, Abes GT. Training of nurses in ear examination and hearing screening in the school setting. Southeast Asian J Trop Med Public Health. 2007;38(1):188-94.

10. Zepillini SL, Bonnafé MC, Pfeifer E. Detecção precoce da deficiência auditiva: um projeto de "capacitação de recursos humanos multiplicadores”. Pró-Fono. 1994;6(1):17-22.

Anexo 1. Referências bibliográficas dos estudos não selecionados para revisão sistemática

\begin{tabular}{|c|c|c|c|}
\hline Ano & Autores & Título & Fonte de publicação \\
\hline 2006 & $\begin{array}{l}\text { Burton SK, Blanton SH, Culpepper B, } \\
\text { White KR, Pandya A, Nance WE, Arnos } \\
\text { KS }\end{array}$ & $\begin{array}{l}\text { Education in the genetics of hearing loss: } \\
\text { a survey of early hearing detection and } \\
\text { intervention programs }\end{array}$ & Genet Med. 2006;8(8):510-7 \\
\hline 2007 & Bally SJ, Bakke MH & $\begin{array}{l}\text { A peer mentor training program for aural } \\
\text { rehabilitation }\end{array}$ & Trends Amplif. 2007;11(2):125-31 \\
\hline 2006 & Olusanya BO, Roberts AA & $\begin{array}{l}\text { Physician education on infant hearing loss in a } \\
\text { developing country }\end{array}$ & Pediatr Rehabil. 2006;9(4):373-7 \\
\hline 2006 & Sataloff RT & $\begin{array}{l}\text { The Council for Accreditation in Occupational } \\
\text { Hearing Conservation: a good model for training } \\
\text { allied health personnel }\end{array}$ & Ear Nose Throat J. 2006;85(5):294-5 \\
\hline 1997 & Cameron $\mathrm{TH}$ & $\begin{array}{l}\text { Epidemiologic issues in audiology: impact on } \\
\text { professional training and service delivery }\end{array}$ & $\begin{array}{l}\text { J Commun Disord. 1997;30(4):285- } \\
\text { 300; quiz 300-1 }\end{array}$ \\
\hline 1997 & Maki-Torkko E, Sorri M, Jarvelin MR & $\begin{array}{l}\text { More education in paediatric audiology needed } \\
\text { for child welfare clinic nurses and doctors }\end{array}$ & Public Health. 1997;111(2):93-6 \\
\hline 1998 & Culpepper NB & $\begin{array}{l}\text { Identifying, training, and supervising personnel } \\
\text { for newborn hearing screening }\end{array}$ & Semin Hear. 1988;19(3):263-71 \\
\hline 1987 & Patterson K, Dancer J & $\begin{array}{l}\text { Alternatives in aural rehabilitation: provider } \\
\text { training of nonaudiologists in the delivery of } \\
\text { hearing-aid supportive services to older persons } \\
\text { with hearing loss }\end{array}$ & Educ Gerontol. 1987;13(6):487-95 \\
\hline 1987 & Wall G, Bukhrer K & $\begin{array}{l}\text { Hearing identification of the preschool child: a } \\
\text { proposed training program }\end{array}$ & $\begin{array}{l}\text { Folia Phoniatr (Basel). } \\
\text { 1987;39(3):145-52 }\end{array}$ \\
\hline 2005 & Proctor R, Niemeyer JA, Compton MV & $\begin{array}{l}\text { Training needs for early intervention personnel } \\
\text { working with infants and toddlers who are deaf } \\
\text { or hard of hearing }\end{array}$ & Volta Review. 2005;105(2):113-28 \\
\hline 1988 & Oyiborhoro JM & Audiology training in Nigeria-I: A training model & Soc Sci Med. 1988;26(10):1035-42 \\
\hline 1988 & Oyiborhoro JM & $\begin{array}{l}\text { Audiology training in Nigeria-II: A cost-effective } \\
\text { approach to training programs }\end{array}$ & Soc Sci Med. 1988;26(10):1043-47 \\
\hline 2006 & $\begin{array}{l}\text { - Olusanya BO, Eletu OB, Odusote O, } \\
\text { Somefun AO, Olude O }\end{array}$ & $\begin{array}{l}\text { Early detection of infant hearing loss: current } \\
\text { experiences of health professionals in a } \\
\text { developing country }\end{array}$ & Acta Paediatr. 2006;95(10):1300-2 \\
\hline
\end{tabular}


Anexo 2. Referências bibliográficas dos estudos selecionados para revisão sistemática

\begin{tabular}{|l|l|l|l|}
\hline Ano & Autores & Título & Fonte de publicação \\
\hline 2001 & McMillan L, Bunning K, Pring T & $\begin{array}{l}\text { The development and evaluation of deaf } \\
\text { awareness training course for support staff }\end{array}$ & $\begin{array}{l}\text { Appl Res Intellect Disabil. } \\
2001 ; 13(4): 283-91\end{array}$ \\
\hline 2001 & $\begin{array}{l}\text { Bennett K, Haggard M, Churchill R, } \\
\text { Wood S }\end{array}$ & $\begin{array}{l}\text { Improving referrals for glue ear from primary } \\
\text { care: are multiple interventions better than one } \\
\text { alone? }\end{array}$ & $\begin{array}{l}\text { J Health Serv Res Policy. } \\
2001 ; 6(3): 139-44\end{array}$ \\
\hline 2004 & Kochkin S & $\begin{array}{l}\text { BHI physician program found to increase use of } \\
\text { hearing healthcare }\end{array}$ & The Hearing Journal. 2004;57(8):27-9. \\
\hline 1994 & $\begin{array}{l}\text { Gloria-Cruz TL, Chiong CM, Chan AL, } \\
\text { Llanes EG, Reyes-Quintos MR, Abes GT }\end{array}$ & $\begin{array}{l}\text { Training of nurses in ear examination and } \\
\text { hearing screening in the school setting }\end{array}$ & $\begin{array}{l}\text { Southeast Asian J Trop Med Public } \\
\text { Health. 2007;38(1):188-94 }\end{array}$ \\
\hline
\end{tabular}

